\title{
A NONLINEAR F-CONTRACTION FORM OF SADOVSKII'S FIXED POINT THEOREM AND ITS APPLIACTION TO A FUNCTIONAL INTEGRAL EQUATION OF VOLTERRA TYPE
}

\author{
Kourosh Nourouzi ${ }^{1}$, Faezeh Zahedi ${ }^{1}$ and Donal O'Regan ${ }^{2}$ \\ 1 Faculty of Mathematics, Tehran, Iran \\ 2 School of Mathematics, Statistics and Applied Mathematics, \\ National University of Ireland, Galway, Ireland
}

\begin{abstract}
In this paper, we give a nonlinear F-contraction form of the Sadovskii fixed point theorem and we also investigate the existence of solutions for a functional integral equation of Volterra type.

Key words: Fixed-point theorems, nonlinear contraction, functional integral equations
\end{abstract}

\section{Introduction and preliminaries}

Let $F$ be an increasing real-valued function on $(0, \infty)$ with $\lim _{t \rightarrow 0} F(t)=-\infty$, and $\varphi:(0, \infty) \rightarrow(0, \infty)$ be a function such that $\liminf _{s \rightarrow t^{+}} \varphi(s)>0$ for any $t \geq 0$. A self-mapping $T$ on a metric space $(X, d)$ is said to be a $(\varphi, F)$-contraction (or a nonlinear $F$-contraction) if

$$
\varphi(d(x, y))+F(d(T x, T y)) \leq F(d(x, y)),
$$

for all $x, y \in X$ provided $T x \neq T y$.

Nonlinear $F$-contractions were considered from a new viewpoint in [13] and the author showed, among other results, that in complete metric spaces these selfmappings have unique fixed points and also under a suitable condition connecting $F$ and $\varphi$, a nonlinear $F$-contraction is condensing with respect to the Hausdorff

\footnotetext{
Received July 17, 2020, accepted November 17, 2020

Communicated by Dijana Mosić

Corresponding Author: Kourosh Nourouzi, Faculty of Mathematics, Tehran, Iran | E-mail: nourouzi@kntu.ac.ir

2010 Mathematics Subject Classification. Primary 47H10; Secondary 47H08, 47N20

(C) 2021 by University of Niš, Serbia | Creative Commons License: CC BY-NC-ND
} 
measure of noncompactness; for more information on nonlinear $F$-contractions see [9] and related references in [13].

The Schauder fixed point theorem states that any continuous self-mapping defined on a nonempty convex and compact subset of a Banach space must have a fixed point and it plays a crucial role in topological fixed point theory. In 1955, Darbo [8] presented a fixed point theorem in terms of the measure of noncompactness (the notion was first defined by Kuratowski [10]) in Banach spaces which generalized the Schauder fixed point theorem. A slight generalization of Darbo's theorem is the Sadovskii fixed point theorem [12]: If $\Omega$ is a nonempty bounded closed convex subset of a Banach $X$ and if $\gamma$ is a measure of noncompactness and $T: \Omega \rightarrow \Omega$ is a continuous mapping satisfying one of the following conditions:

(1) (Darbo [8]) $T$ is a $k$-set contraction, i.e., there exists $k \in[0,1)$ such that for any set $C \subset \Omega$,

$$
\gamma(T(C)) \leq k \gamma(C) ;
$$

(2) (Sadovskii [12]) $T$ is a $\gamma$-condensing mapping, i.e., for any set $C \subset \Omega$ with positive measure of noncompactness,

$$
\gamma(T(C))<\gamma(C),
$$

then $T$ has a fixed point.

Some generalizations of these fixed point theorems can be found for example in $[1,7,11]$ and the references therein.

In this paper, we give a nonlinear $F$-contraction form of the Sadovskii fixed point theorem (Theorem 1.1) and then inspired by the main result in [13] (and [1]) we give an application to solving a functional integral equation of Volterra type (Theorem 2.1).

We first give some preliminaries which will be needed in this paper.

Measures of noncompactness serve as useful tools in the theory of operator equations in Banach spaces and are used in the theory of functional equations, ordinary and partial differential equations, integral and integro-differential equations, etc (see e.g., $[2,3,4,5]$ ). In this section, we give a generalization of the Sadovskii fixed point theorem in terms of a general notion of the measure of noncompactness and nonlinear $F$-contractions.

For the convenience of the reader we recall some basic notations and definitions; see $[2,3,4,5]$. Let $\mathbb{R}$ denote the set of all real numbers and $\mathbb{R}_{0}^{+}=[0, \infty)$. In a Banach space $E$, the symbols $\bar{X}$ and conv $X$ stand for the closure and closed convex hull of the subset $X$ of $E$, respectively. Denote by $\mathfrak{M}_{E}$ the family of all nonempty bounded subsets of $E$ and by $\mathfrak{N}_{E}$ the family consisting of all nonempty relatively compact subsets of $E$.

Definition 1.1. A measure of noncompactness in the Banach space $E$ is a mapping $\mu: \mathfrak{M}_{E} \rightarrow \mathbb{R}_{0}^{+}$which satisfies the following conditions:

(MN1) the family $\operatorname{ker} \mu=\left\{X \in \mathfrak{M}_{E}: \mu(X)=0\right\}$ is nonempty and ker $\mu \subset \mathfrak{N}_{E}$; (MN2) $\mu(X) \leq \mu(Y)$ if $X \subset Y$; 
(MN3) $\mu(X)=\mu(\bar{X})$, for all $X \in \mathfrak{M}_{E}$;

(MN4) $\mu(X)=\mu(\operatorname{conv} X)$, for all $X \in \mathfrak{M}_{E}$;

(MN5) $\mu(t X+(1-t) Y) \leq t \mu(X)+(1-t) \mu(Y)$, for all $X, Y \in \mathfrak{M}_{E}$ and $t \in[0,1]$;

(MN6) If $\left\{X_{n}\right\}$ is a decreasing sequence of closed and nonempty sets of $\mathfrak{M}_{E}$ with $\lim _{n \rightarrow \infty} \mu\left(X_{n}\right)=0$, then $\cap_{n=1}^{\infty} X_{n}$ is nonempty.

The following result is a generalization of the Sadovskii fixed point theorem using $F$-contractions.

Theorem 1.1. Let $\Omega$ be a nonempty bounded closed convex subset of a Banach space $E$ and $T: \Omega \rightarrow \Omega$ be a continuous mapping which satisfies

$$
\varphi(\mu(X))+F(\mu(T X)) \leq F(\mu(X)),
$$

for any nonempty subset $X$ of $\Omega$ with $\mu(T X) \neq 0$ and $\mu(X) \neq 0$, where $\mu$ is an arbitrary measure of noncompactness and $F:(0, \infty) \rightarrow \mathbb{R}$ and $\varphi:(0, \infty) \rightarrow(0, \infty)$ are functions such that

$$
\forall\left(t_{n}\right) \in(0, \infty)^{\mathbb{N}}\left(F\left(t_{n}\right) \rightarrow-\infty \Rightarrow t_{n} \rightarrow 0\right)
$$

and

$$
\liminf _{s \rightarrow t^{+}} \varphi(s)>0, \quad(t \geq 0)
$$

Then $T$ has a fixed point.

Proof. Define a sequence $\left(\Omega_{n}\right)$ inductively as follows:

$$
\Omega_{0}=\Omega \quad \text { and } \quad \Omega_{n}=\operatorname{conv} T \Omega_{n-1}, \quad n \geq 1 .
$$

We may assume, without loss of generality, that $\mu\left(\Omega_{n}\right)>0$, for every $n \geq 1$, for otherwise, by Schauder's fixed point theorem $T$ has a fixed point.

Observe that $\Omega_{n+1} \subset \Omega_{n}$ for all $n \geq 1$, which means that the sequence $\left(\mu\left(\Omega_{n}\right)\right)$ is decreasing and therefore is convergent to some nonnegative real number. From (1.2), there exist a $c>0$ and $n_{1} \in \mathbb{N}$ such that $\varphi\left(\mu\left(\Omega_{n}\right)\right)>c$ for all $n \geq n_{1}$. Therefore, we have

$$
\begin{aligned}
F\left(\mu\left(\Omega_{n}\right)\right) & \leq F\left(\mu\left(\Omega_{n-1}\right)\right)-\varphi\left(\mu\left(\Omega_{n-1}\right)\right) \\
& \vdots \\
& \leq F\left(\mu\left(\Omega_{0}\right)\right)-\sum_{i=0}^{n-1} \varphi\left(\mu\left(\Omega_{i}\right)\right) \\
& =F\left(\mu\left(\Omega_{0}\right)\right)-\sum_{i=0}^{n_{1}-1} \varphi\left(\mu\left(\Omega_{i}\right)\right)-\sum_{i=n_{1}}^{n-1} \varphi\left(\mu\left(\Omega_{i}\right)\right) \\
& <F\left(\mu\left(\Omega_{0}\right)\right)-\left(n-n_{1}\right) c,
\end{aligned}
$$


for all $n>n_{1}$. We get $F\left(\mu\left(\Omega_{n}\right)\right) \rightarrow-\infty$ as $n \rightarrow \infty$ and hence by (1.1), $\mu\left(\Omega_{n}\right) \rightarrow 0$.

Since $\left(\Omega_{n}\right)$ is a decreasing sequence of closed and nonempty sets of $\mathfrak{M}_{E}$ with $\lim _{n \rightarrow \infty} \mu\left(\Omega_{n}\right)=0$, by (MN1) and (MN6) of Definition 1.1 we have that $\Omega_{\infty}=$ $\cap_{n=0}^{\infty} \Omega_{n}$ is a nonempty compact convex subset of $\Omega$. Moreover, $T$ maps $\Omega_{\infty}$ into itself. Now, applying the Schauder fixed point theorem we infer that $T$ has a fixed point in $\Omega_{\infty}$.

Note that in the setting of Banach spaces Theorem 2.1 in [13] can be deduced from Theorem 1.1. Furthermore, notice that putting $F(x)=\ln x$ in Theorem 1.1 we have the Sadovskii fixed point theorem. In fact, we have

$$
\varphi(\mu(X))+\ln (\mu(T X)) \leq \ln (\mu(X))
$$

and therefore

$$
\mu(T X) \leq \frac{1}{e^{\varphi(\mu(X))}} \mu(X)<\mu(X) .
$$

Also, Theorem 1.1 can be easily generalized in the spirit of [11, Theorem 5]. Indeed, let $\Phi$ denote the set of all functions $f: \mathbb{R}^{+^{2}} \rightarrow \mathbb{R}$ satisfying

$$
\forall\left(t_{n}\right) \in(0, \infty)^{\mathbb{N}}\left(\forall n \in \mathbb{N}\left(t_{n+1} \leq t_{n} \wedge f\left(t_{n+1}, t_{n}\right) \geq 0\right) \Rightarrow t_{n} \rightarrow 0\right) .
$$

Observe that $f \in \Phi$ such that $f(x, y)=-F(x)+F(y)-\varphi(y)$, where $F$ and $\varphi$ satisfy (1.1) and (1.2), respectively. Then, we clearly have the following:

Theorem 1.2. Let $\mu$ be a measure of noncompactness on a Banach space E. Assume that $\Omega$ is a nonempty bounded closed and convex subset of $E$ and $T: \Omega \rightarrow \Omega$ is a continuous mapping such that

$$
\exists f \in \Phi \forall X \subset \Omega(\bar{X} \text { is noncompact } \Rightarrow f(\mu(T X), \mu(X)) \geq 0) .
$$

Then, $T$ has a fixed point.

\section{Application to a functional integral equation of Volterra type}

In this section, we follow the terminology and notations used in [6] unless otherwise specified. Consider the Banach space $B C\left(\mathbb{R}_{0}^{+}\right)$consisting of all bounded and continuous real-valued functions on the nonnegative real numbers $\mathbb{R}_{0}^{+}$equipped with the norm

$$
\|x\|=\sup \{|x(t)|: t \geq 0\},
$$

where $x \in B C\left(\mathbb{R}_{0}^{+}\right)$. Let $X$ be a nonempty bounded subset of $B C\left(\mathbb{R}_{0}^{+}\right)$and $L>0$. For $x \in X$ and $\varepsilon>0$, the modules of continuity of the function $x$ on the interval $[0, L]$, denoted by $w^{L}(x, \varepsilon)$, is defined as

$$
w^{L}(x, \varepsilon)=\sup \{|x(t)-x(s)|: t, s \in[0, L],|t-s| \leq \varepsilon\} .
$$


Moreover, let

$$
\begin{aligned}
w^{L}(X, \varepsilon) & =\sup \left\{w^{L}(x, \varepsilon): x \in X\right\} \\
w_{0}^{L}(X) & =\lim _{\varepsilon \rightarrow 0} w^{L}(X, \varepsilon) \\
w_{0}(X) & =\lim _{L \rightarrow \infty} w_{0}^{L}(X) .
\end{aligned}
$$

Also, for a fixed number $t \in \mathbb{R}_{0}^{+}$let

$$
X(t)=\{x(t): x \in X\} .
$$

Define a function $\mu$ on the family $\mathfrak{M}_{B C\left(\mathbb{R}_{0}^{+}\right)}$as

$$
\mu(X)=w_{0}(X)+\limsup _{t \rightarrow \infty} \operatorname{diam} X(t),
$$

where diam $X(t)$ is defined as

$$
\operatorname{diam} X(t)=\sup \{|x(t)-y(t)|: x, y \in X\} .
$$

The function $\mu$ defined above is a measure of noncompactness in the Banach space $B C\left(\mathbb{R}_{0}^{+}\right)$(see $\left.[4,6]\right)$.

Let $\mathcal{F}$ denote the set of all functions $F:(0, \infty) \rightarrow \mathbb{R}$ which satisfy the following conditions:

(F1) For all $t_{1}, t_{2}>0, t_{1}>t_{2}$ implies $F\left(t_{1}\right)>F\left(t_{2}\right)$;

(F2) For any sequence $\left(t_{n}\right) \subset(0, \infty), t_{n} \rightarrow 0$ if and only if $F\left(t_{n}\right) \rightarrow-\infty$.

Suppose that two functions $f$ and $g$ satisfy the following conditions:

(i) $f: \mathbb{R}_{0}^{+} \times \mathbb{R} \rightarrow \mathbb{R}$ is a continuous function such that $t \mapsto f(t, 0)$ is also an element of the Banach space $B C\left(\mathbb{R}_{0}^{+}\right), f(0,0)=0$, and $c \leq f(0,2 c)$ for each $c \geq 0$;

(ii) If $F \in \mathcal{F}$ is continuous and $\varphi:(0, \infty) \rightarrow(0, \infty)$ is a nonincreasing continuous function such that

$$
\lim _{s \rightarrow t^{+}} \varphi(s)>0, \quad\left(t \in \mathbb{R}_{0}^{+}\right)
$$

(or equivalently, $\liminf _{s \rightarrow t^{+}} \varphi(s)>0$ ), then

$F\left(\left|f(s, u)-f\left(s, u_{1}\right)\right|+\left|f(t, w)-f\left(t, w_{1}\right)\right|+f(0, c)\right) \leq(F-\varphi)\left(\left|u-u_{1}\right|+\left|w-w_{1}\right|+c\right)$,

where $c, s, t \in \mathbb{R}_{0}^{+}$and $u, u_{1}, w, w_{1} \in \mathbb{R}$ with

$\left|f(s, u)-f\left(s, u_{1}\right)\right|+\left|f(t, w)-f\left(t, w_{1}\right)\right|+f(0, c)>0$ and $\left|u-u_{1}\right|+\left|w-w_{1}\right|+c>0$.

Note that under the above assumptions we, in particular, have

$$
F\left(\left|f(s, u)-f\left(s, u_{1}\right)\right|\right)<F\left(\left|u-u_{1}\right|\right)
$$


and consequently

$$
\mid f(s, u)-f\left(s, u_{1}|\leq| u-u_{1} \mid\right.
$$

(iii) $g: \mathbb{R}_{0}^{+} \times \mathbb{R}_{0}^{+} \times \mathbb{R} \rightarrow \mathbb{R}$ is a continuous function and there exist continuous functions $\alpha, \beta: \mathbb{R}_{0}^{+} \rightarrow \mathbb{R}_{0}^{+}$such that

$$
\lim _{t \rightarrow \infty} \alpha(t) \int_{0}^{t} \beta(s) d s=0
$$

and

$$
|g(t, s, x)| \leq \alpha(t) \beta(s)
$$

for any $x \in \mathbb{R}$ and $t, s \in \mathbb{R}_{0}^{+}$with $s \leq t$;

(iv) With

$$
\kappa=\sup \left\{f(t, 0)+\alpha(t) \int_{0}^{t} \beta(s) d s: t \geq 0\right\},
$$

we have $0 \leq \kappa<\infty$ and there exists a positive real number $r_{0}$ such that

$$
(F-\varphi)(r+2 \kappa) \leq F(r) .
$$

Example 2.1. The following functions satisfy conditions (i)-(iv):

- $f: \mathbb{R}_{0}^{+} \times \mathbb{R} \rightarrow \mathbb{R}$ defined by $f(s, t)=|\sin (s)|+\frac{t}{2} ;$

- $F \in \mathcal{F}$ defined by $F(t)=-\frac{1}{t}$;

- $\varphi:(0, \infty) \rightarrow(0, \infty)$ defined by $\varphi(t)=\frac{1}{t}$;

- $g: \mathbb{R}_{0}^{+} \times \mathbb{R}_{0}^{+} \times \mathbb{R} \rightarrow \mathbb{R}$ defined by $g(t, s, x)=s e^{-t} \frac{h_{1}(x)}{1+\left|h_{2}(x)\right|}$, where $h_{1}, h_{2} \in$ $B C(\mathbb{R})$ with $\left|h_{1}(x)\right| \leq 1+\left|h_{2}(x)\right|$, for all $x \in \mathbb{R}$;

- $\alpha, \beta: \mathbb{R}_{0}^{+} \rightarrow \mathbb{R}_{0}^{+}$defined by $\alpha(t)=e^{-t}, \beta(s)=s$.

Now we give an application of Theorem 1.1 where we show that a functional equation of Volterra type has a solution.

Theorem 2.1. The integral equation of Volterra type

$$
x(t)=f(t, x(t))+\int_{0}^{t} g(t, s, x(s)) d s, \quad\left(t \in \mathbb{R}_{0}^{+}\right)
$$

where $f$ and $g$ are functions satisfying conditions (i)-(iv) has a solution in the Banach space $B C\left(\mathbb{R}_{0}^{+}\right)$. 
Proof. We will use some ideas from [6]. Define a mapping $T$ on the Banach space $B C\left(\mathbb{R}_{0}^{+}\right)$as follows:

$$
(T x)(t)=f(t, x(t))+\int_{0}^{t} g(t, s, x(s)) d s, \quad\left(t \in \mathbb{R}_{0}^{+}\right) .
$$

It is easy to see that for any $x \in B C\left(\mathbb{R}_{0}^{+}\right)$, the function $T x$ is a real-valued continuous function on $\mathbb{R}_{0}^{+}$. In addition, for any function $x \in B C\left(\mathbb{R}_{0}^{+}\right)$and $t \geq 0$ we have

$$
\begin{aligned}
F(|(T x)(t)|) & =F\left(\left|f(t, x(t))+\int_{0}^{t} g(t, s, x(s)) d s\right|\right) \\
& \leq F\left(|f(t, x(t))-f(t, 0)|+\int_{0}^{t}|g(t, s, x(s))| d s+f(t, 0)\right) \\
& \leq F(|f(t, x(t))-f(t, 0)|+\kappa) \\
& \leq F(|f(t, x(t))-f(t, 0)|+f(0,2 \kappa)) \\
& \leq(F-\varphi)(\mid x(t)) \mid+2 \kappa) \\
& \leq(F-\varphi)(\|x\|+2 \kappa) .
\end{aligned}
$$

From assumption (iv), choose $r_{0}>0$ such that $(F-\varphi)\left(r_{0}+2 \kappa\right) \leq F\left(r_{0}\right)$. Then, in particular, we have

$F(|(T x)(t)|) \leq(F-\varphi)(|x(t)|+2 \kappa) \leq(F-\varphi)(\|x\|+2 \kappa) \leq(F-\varphi)\left(r_{0}+2 \kappa\right) \leq F\left(r_{0}\right)$,

when $\|x\| \leq r_{0}$ and since $F$ is increasing we get $|(T x)(t)| \leq r_{0}$ and finally since $t \geq 0$ was arbitrary, we have $\|T x\| \leq r_{0}$. Therefore $T$ maps the closed ball $B_{r_{0}}=$ $\left\{x \in B C\left(\mathbb{R}_{0}^{+}\right):\|x\| \leq r_{0}\right\}$ into itself. (Notice also, since

$$
F(|(T x)(t)|) \leq F(|f(t, x(t))-f(t, 0)|+\kappa) \leq F(\mid x(t)) \mid+\kappa) \leq F(\|x\|+\kappa),
$$

$T x$ is bounded for any $x \in B C\left(\mathbb{R}_{0}^{+}\right)$.)

Now, we show that the self-mapping $T$ is continuous on the ball $B_{r_{0}}$. Let $\varepsilon>0$ be given. Suppose that $x, y \in B_{r_{0}}$ such that $\|x-y\|<\varepsilon$. We have

$$
\begin{aligned}
F & (|(T x)(t)-(T y)(t)|) \\
& \leq F\left(|f(t, x(t))-f(t, y(t))|+\left|\int_{0}^{t} g(t, s, x(s)) d s-\int_{0}^{t} g(t, s, y(s)) d s\right|\right) \\
& \leq F\left(|f(t, x(t))-f(t, y(t))|+\left|\int_{0}^{t} g(t, s, x(s)) d s\right|+\left|\int_{0}^{t} g(t, s, y(s)) d s\right|\right) \\
& \leq F\left(|f(t, x(t))-f(t, y(t))|+2 \alpha(t) \int_{0}^{t} \beta(s) d s\right),
\end{aligned}
$$

for any $t \geq 0$. From assumption (iii), there exists a number $L>0$ such that

$$
2 \alpha(t) \int_{0}^{t} \beta(s) d s<\varepsilon
$$


for each $t \geq L$. Thus, for an arbitrary $t \geq L$ we obtain

$$
\begin{aligned}
F(|(T x)(t)-(T y)(t)|) & \leq F(|f(t, x(t))-f(t, y(t))|+\varepsilon) \\
& \leq F(\mid x(t))-y(t)) \mid+\varepsilon) \\
& <F(2 \varepsilon)
\end{aligned}
$$

and since $F$ is increasing, we obtain

$$
|(T x)(t)-(T y)(t)| \leq 2 \varepsilon, \quad(t \geq L) .
$$

Since the function $g$ is uniformly continuous on the set $[0, L] \times[0, L] \times\left[-r_{0}, r_{0}\right]$, we have $\lim _{\varepsilon \rightarrow 0} w^{L}(g, \varepsilon)=0$, where

$$
w^{L}(g, \varepsilon)=\sup \left\{\left|g(t, s, x)-g(t, s, y): t, s \in[0, L], x, y \in\left[-r_{0}, r_{0}\right],\right| x-y \mid \leq \varepsilon\right\} .
$$

On the other hand, for an arbitrary fixed $t \in[0, L]$, we have

$$
\begin{aligned}
F & (|(T x)(t)-(T y)(t)|) \\
& \leq F\left(|f(t, x(t))-f(t, y(t))|+\left|\int_{0}^{t} g(t, s, x(s)) d s-\int_{0}^{t} g(t, s, y(s)) d s\right|\right) \\
& \leq F\left(|f(t, x(t))-f(t, y(t))|+\int_{0}^{L} w^{L}(g, \varepsilon) d s\right) \\
& =F\left(|f(t, x(t))-f(t, y(t))|+L w^{L}(g, \varepsilon)\right) \\
& \leq F\left(|x(t)-y(t)|+L w^{L}(g, \varepsilon)\right),
\end{aligned}
$$

and since $F$ is increasing we obtain

$$
|(T x)(t)-(T y)(t)| \leq|x(t)-y(t)|+L w^{L}(g, \varepsilon),
$$

for any $t \in[0, L]$. Finally, the continuity of $T$ on the ball $B_{r_{0}}$ is obtained from (2.4) and (2.5).

Let $X$ be an arbitrary nonempty subset of the closed ball $B_{r_{0}}$. Let $\varepsilon>0, L>0$, $z, w \in X$, and $t^{\prime}>0$ be fixed. Choose $t, s \in[0, L]$ with $|t-s| \leq \varepsilon$. Without loss of generality, we assume that $s<t$. Then for any $x, y \in X$ we have

$$
\begin{aligned}
F(\mid & \left.(T x)(t)-(T x)(s)|+|(T z)\left(t^{\prime}\right)-(T w)\left(t^{\prime}\right) \mid\right) \\
\leq & F\left(\left|f(t, x(t))+\int_{0}^{t} g(t, \tau, x(\tau)) d \tau-f(s, x(s))-\int_{0}^{s} g(s, \tau, x(\tau)) d \tau\right|\right. \\
& \left.+\left|f\left(t^{\prime}, z\left(t^{\prime}\right)\right)+\int_{0}^{t^{\prime}} g\left(t^{\prime}, s, z(s)\right) d s-f\left(t^{\prime}, w\left(t^{\prime}\right)\right)-\int_{0}^{t^{\prime}} g\left(t^{\prime}, s, w(s)\right) d s\right|\right) \\
\leq & F\left(|f(t, x(t))-f(s, x(t))|+|f(s, x(t))-f(s, x(s))|+\mid \int_{0}^{t} g(t, \tau, x(\tau)) d \tau\right. \\
& -\int_{0}^{t} g(s, \tau, x(\tau)) d \tau|+| \int_{s}^{t} g(s, \tau, x(\tau)) d \tau|+| f\left(t^{\prime}, z\left(t^{\prime}\right)\right)-f\left(t^{\prime}, w\left(t^{\prime}\right)\right) \mid \\
& \left.+\left|\int_{0}^{t^{\prime}} g\left(t^{\prime}, s, z(s)\right) d s-\int_{0}^{t^{\prime}} g\left(t^{\prime}, s, w(s)\right) d s\right|\right) \\
\leq & F\left(w_{1}^{L}(f, \varepsilon)+|f(s, x(t))-f(s, x(s))|+L w_{1}^{L}(g, \varepsilon)+\int_{s}^{t}|g(t, \tau, x(\tau))| d \tau\right. \\
& \left.+\left|f\left(t^{\prime}, z\left(t^{\prime}\right)\right)-f\left(t^{\prime}, w\left(t^{\prime}\right)\right)\right|+\int_{0}^{t^{\prime}}\left|g\left(t^{\prime}, s, z(s)\right)-g\left(t^{\prime}, s, w(s)\right)\right| d s\right) \\
\leq & F\left(w_{1}^{L}(f, \varepsilon)+|f(t, x(t))-f(s, x(s))|+L w_{1}^{L}(g, \varepsilon)+\varepsilon \sup \{\alpha(s) \beta(t): t, s \in[0, L]\}\right. \\
& \left.\left.\left.+\mid f\left(t^{\prime}, z\left(t^{\prime}\right)\right)-f\left(t^{\prime}, w\left(t^{\prime}\right)\right)\right) \mid+2 \alpha\left(t^{\prime}\right) \int_{0}^{t^{\prime}} \beta(s) d s\right)\right)
\end{aligned}
$$


$\leq F\left(|f(t, x(t))-f(s, x(s))|+\left|f\left(t^{\prime}, z\left(t^{\prime}\right)\right)-f\left(t^{\prime}, w\left(t^{\prime}\right)\right)\right|+\eta_{f, g}^{L}(\varepsilon)+\gamma\left(t^{\prime}\right)\right)$,

where

$$
\begin{gathered}
w_{1}^{L}(f, \varepsilon)=\sup \left\{|f(t, x)-f(s, x)|: t, s \in[0, L], x \in\left[-r_{0}, r_{0}\right],|t-s| \leq \varepsilon\right\} \\
w_{1}^{L}(g, \varepsilon)=\sup \left\{|g(t, \tau, x)-g(s, \tau, x)|: t, s, \tau \in[0, L], x \in\left[-r_{0}, r_{0}\right],|t-s| \leq \varepsilon\right\},
\end{gathered}
$$

and

$$
\begin{gathered}
\eta_{f, g}^{L}(\varepsilon)=w_{1}^{L}(f, \varepsilon)+L w_{1}^{L}(g, \varepsilon)+\varepsilon \sup \{\alpha(s) \beta(t): t, s \in[0, L]\} \\
\gamma\left(t^{\prime}\right)=2 \alpha\left(t^{\prime}\right) \int_{0}^{t^{\prime}} \beta(s) d s .
\end{gathered}
$$

Notice that since $f$ and $g$ are uniformly continuous on the sets $[0, L] \times\left[-r_{0}, r_{0}\right]$ and $[0, L] \times[0, L] \times\left[-r_{0}, r_{0}\right]$, respectively, we have $w_{1}^{L}(f, \varepsilon) \rightarrow 0$ and $w_{1}^{L}(g, \varepsilon) \rightarrow 0$ as $\varepsilon \rightarrow 0$. In addition, since $\alpha$ and $\beta$ are continuous functions on $\mathbb{R}_{0}^{+}$, we have

$$
\sup \{\alpha(s) \beta(t): t, s \in[0, L]\}<\infty .
$$

Note also that, from (2.2), we have $\gamma\left(t^{\prime}\right) \rightarrow 0$ as $t^{\prime} \rightarrow \infty$. Since, from assumption (iv),

$$
\left.\eta_{f, g}^{L}(\varepsilon)+\gamma\left(t^{\prime}\right)\right) \leq f\left(0,2\left(\eta_{f, g}^{L}(\varepsilon)+\gamma\left(t^{\prime}\right)\right)\right),
$$

using assumption (iii) we obtain

$$
\begin{aligned}
& F\left(|(T x)(t)-(T x)(s)|+\left|(T z)\left(t^{\prime}\right)-(T w)\left(t^{\prime}\right)\right|\right) \\
& \quad \leq(F-\varphi)\left(|x(t)-x(s)|+\left|z\left(t^{\prime}\right)-w\left(t^{\prime}\right)\right|+2 \eta_{f, g}^{L}(\varepsilon)+2 \gamma\left(t^{\prime}\right)\right) .
\end{aligned}
$$

Now, taking the supremum of the previous inequality as $t, s \in[0, L]$ with $|t-s| \leq \varepsilon$ we get

$$
\begin{aligned}
& F\left(w^{L}(T x, \varepsilon)+\left|(T z)\left(t^{\prime}\right)-(T w)\left(t^{\prime}\right)\right|\right) \\
& \left.\quad \leq(F-\varphi)\left(w^{L}(x, \varepsilon)\right)+\left|z\left(t^{\prime}\right)-w\left(t^{\prime}\right)\right|+2 \eta_{f, g}^{L}(\varepsilon)+2 \gamma\left(t^{\prime}\right)\right) .
\end{aligned}
$$

Taking the supremum as $z, w \in X$, we have

$\left.F\left(w^{L}(T X, \varepsilon)+\operatorname{diam}(T X)\left(t^{\prime}\right)\right) \leq(F-\varphi)\left(w^{L}(X, \varepsilon)\right)+\operatorname{diam} X\left(t^{\prime}\right)+2 \eta_{f, g}^{L}(\varepsilon)+2 \gamma\left(t^{\prime}\right)\right)$.

Letting first $\varepsilon \rightarrow 0$ and then $L \rightarrow \infty$ we get

$$
F\left(w_{0}(T X)+\operatorname{diam}(T X)\left(t^{\prime}\right)\right) \leq(F-\varphi)\left(w_{0}(X)+\operatorname{diam} X\left(t^{\prime}\right)+2 \gamma\left(t^{\prime}\right)\right) .
$$

Finally, taking the limit superior as $t^{\prime} \rightarrow \infty$ we obtain

$$
F\left(w_{0}(T X)+\limsup _{t^{\prime} \rightarrow \infty} \operatorname{diam}(T X)\left(t^{\prime}\right)\right) \leq(F-\varphi)\left(w_{0}(X)+\limsup _{t^{\prime} \rightarrow \infty} \operatorname{diam} X\left(t^{\prime}\right)\right) .
$$


This can be restated as

$$
F(\mu(T X)) \leq F(\mu(X))-\varphi(\mu(X)),
$$

in which $\mu$ is the measure of noncompactness given in (2.1). Now, Theorem 1.1 gives the desired result.

Example 2.2. Consider the functions given in Example 2.1. An easy application of Theorem 2.1 shows that the integral equation

$$
x(t)=2|\sin (t)|+\int_{0}^{t} \frac{2 s e^{-t} \sin ^{3} x(s)}{1+\cos ^{2} x(s)} d s, \quad\left(t \in \mathbb{R}_{0}^{+}\right)
$$

has a solution in the Banach space $B C\left(\mathbb{R}_{0}^{+}\right)$.

\section{Acknowledgments}

The authors would like to thank the anonymous referee for constructive comments to improve the paper.

\section{REF E R E N C E S}

1. A. Aghajani, J. Banaś and N. Sabzali: Some generalizations of Darbo fixed point theorem and applications. Bull. Belg. Math. Soc. Simon Stevin 20 (2013), 345-358.

2. R. R. Akmerov, M. I. Kamenski, A. S. Potapov, A. E. Rodkina and B. N. SADOVSKII: Measure of noncompactness and condensing operators. Birkhavervser Verlag, Basel 1992.

3. J. M. Ayerbe Toledano, T. Domínguez Benavides and G. López Acedo: Measures of noncompactness in metric fixed point theory. Operator Theory: Advances and Applications, 99. Birkhuser Verlag, Basel, 1997.

4. J. BAnAś and K. GoBel: Measures of noncompactness in Banach spaces. Lect. Notes Pure Appl. Math., vol. 60, Dekker, New York, 1980.

5. J. Banaś, M. Jleli, M. Mursaleen, B. Samet and C. Vetro: Advances in nonlinear analysis via the concept of measure of noncompactness. Springer, Singapore, 2017.

6. J. BANAŚ and B. RzEPKA: An application of a measure of noncompactness in the study of asymptotic stability. Appl. Math. Letters 16 (2003), 1-6.

7. J. Garcia-FAlSet and K. LATraCh: On Darbo-Sadovskii's fixed point theorems type for abstract measures of (weak) noncompactness. Bull. Belg. Math. Soc. Simon Stevin, 22 (2015), no. 5, 797-812.

8. G. Darbo: Punti uniti in transformaziationi a condominio non comatto, Rend. Sem. Mat. Univ. Padova. 24 (1955), 84-92.

9. D. KLIM and D. WARDOWSKI: Fixed points of dynamic processes of set-valued F-contractions and application to functional equations, Fixed Point Theory Appl., 2015:22, 9 pp. 
10. K. Kuratowski: Sur les espaces complets, Fundam. Math. 5 (1930), 301-309.

11. R. Pietkun: On some generalizations of the Mönch, Sadovskii and Darbo fixed point theorems. J. Fixed Point Theory Appl. 20 (2018), no. 3, Art. 95, 11 pp.

12. B. N. SAdovskir: A fixed-point principle, Funct. Anal. Appl. 1 (1967), no. 2, 151-153.

13. D. Wardowski: Solving existence problems via F-contractions, Proc. Amer. Math. Soc. 146 (2018), no. 4, 1585-1598. 